\title{
Synergistic antitumor effect of the combination of a dual cancer-specific oncolytic adenovirus and cisplatin on lung cancer cells
}

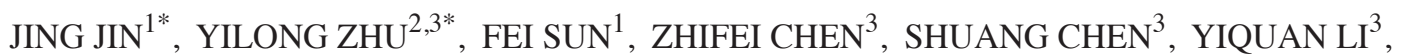 \\ WENJIE $\mathrm{LI}^{3}, \mathrm{MIN} \mathrm{LI}^{3}, \mathrm{CHUANXIN} \mathrm{CUI}^{3}$, YINGLI CUI ${ }^{3}, \mathrm{XUNZHI} \mathrm{YIN}^{3}, \mathrm{SHANZHI} \mathrm{LI}^{3}$, \\ JIN ZHAO ${ }^{3}$, GUO YAN ${ }^{2}$, XIAO LI $^{1-3}$ and NINGYI JIN ${ }^{1-3}$ \\ ${ }^{1}$ Institute of Frontier Medical Science, Jilin University, Changchun, Jilin 130021; \\ ${ }^{2}$ Academicians Workstation of Jilin Province, Changchun University of Chinese Medicine, Changchun, Jilin 130117; \\ ${ }^{3}$ Key Laboratory of Jilin Province for Zoonosis Prevention and Control, Institute of Military Veterinary Medicine, \\ Academy of Military Medical Sciences, Changchun, Jilin 130122, P.R. China
}

Received February 1, 2018; Accepted April 26, 2018

DOI: $10.3892 / \mathrm{ol} .2018 .9470$

\begin{abstract}
The effect of the combination of a recombinant adenovirus (ATV) expressing a specific apoptin protein and cisplatin on human lung cancer cells (A549 cells) was determined. The inhibitory effects of ATV and cisplatin, ATV alone, or cisplatin alone on the migration and invasion of A549 cells were evaluated in vitro using cell proliferation, wound healing, Transwell migration and Matrigel invasion assays. The tumor inhibition effect on A549 cells in vivo was assessed by observing the tumor growth and survival rate of nude mice with subcutaneous tumor xenografts grown from implanted A549 cells after treatment with ATV, cisplatin, or ATV combined with cisplatin. The proliferation $(\mathrm{P}<0.01)$, migration $(\mathrm{P}<0.01)$, and invasion $(\mathrm{P}<0.01)$ on $\mathrm{A} 549$ cells was suppressed significantly by ATV, cisplatin, and ATV and cisplatin, in a dose- and time-dependent manner. The inhibition of tumor growth in transplanted nude mice in the ATV combined with cisplatin group was significantly higher than that displayed in the other groups, and the survival rate of the combined treatment group was significantly higher than that of the group treated with cisplatin alone. The results indicated that the combined application of ATV and cisplatin could reduce toxicity and showed a synergistic effect in reducing
\end{abstract}

Correspondence to: Professor Ningyi Jin or Professor Xiao Li, Key Laboratory of Jilin Province for Zoonosis Prevention and Control, Institute of Military Veterinary Medicine, Academy of Military Medical Sciences, 666 LiuYing Xi Road, Changchun, Jilin 130122, P.R. China

E-mail: ningyij@hotmail.com

E-mail: skylee6226@163.com

*Contributed equally

Key words: oncolytic adenovirus, cisplatin, tumor inhibition, toxicity tumor growth and increasing survival. Thus, there is a potential research value in treating tumors using the combination of ATV and cisplatin, which provides a foundation for future preclinical studies on this antitumor treatment.

\section{Introduction}

The results from the National Central Cancer Registry (NCCR) collected registration data in 2013 from local cancer registries (1) showed that the incidence and mortality rates of lung cancer occupied the first position among malignant tumors, and there are about 733,000 new cases and 591,000 deaths each year in China. Platinum-based therapy, which is used for clinical tumor treatment in the chemotherapeutic regimen in China, was used in $80 \%$ of cases (2). Cisplatin is one of the most common platinum drugs; however, tumor drug-resistance (3), nephrotoxicity $(4,5)$, hepatotoxicity (6), ototoxicity (7), and other side-effects are associated with the long-term use of cisplatin. In addition, advanced lung cancers are difficult to treat with the currently available platinum-based chemotherapeutic regimens. Therefore, novel therapeutic regimens that combine cisplatin-based therapy with other methods to reduce side effects and increase the curative effect are urgently needed.

The apoptin protein from chicken anemia virus (CAV) exhibits selective cytotoxicity, which is enabled by its phosphorylation and translocation to the nucleus for tumor cells without affecting normal diploid cells (8). Apoptin aggregates mainly in the cancer cell nucleus where it plays a role in specific cytotoxicity, while it is mainly localized in the cytoplasm of normal cells where it is degraded (9). The size of xenografted tumors in mice could be reduced effectively by various delivery strategies of apoptin (10-12). However, an effective cancer treatment strategy also needs a safe delivery vehicle that can express apoptin protein persistently and deliver it effectively. The human adenovirus serotype 5 (Ad5) is one of such vector that has been used widely to treat a variety of tumors (13-16). In addition, satisfactory curative effects in the 
treatment of tumors have been demonstrated by a combination of a recombinant Ad5 and cisplatin $(17,18)$.

A dual cancer-specific oncolytic adenovirus ATV containing the apoptin gene (with the capacity for tumor specific cytotoxicity) under the control of the hTERTp promoter (enabling tumor-dependent, specific replication) was constructed in our laboratory (19-23). In the present study, we used the combination of ATV and cisplatin to inhibit tumor formation, and the migration and invasion of A549 cells. The effects were assessed using cell proliferation assays, wound healing assays, Transwell migration assays, Matrigel invasion assay, and nude mouse models with subcutaneous tumor xenografts of A549 cells.

\section{Materials and methods}

Virus, cell lines, reagents and mice. ATV was constructed previously in our laboratory. Human lung adenocarcinoma (A549) cells were purchased from the Cell Bank, Type Culture Collection of the Chinese Academy of Sciences (Shanghai, China). Hank's F12 medium, Dulbecco's modified Eagle's medium (DMEM), fetal bovine serum, and $0.25 \%$ trypsin solution were purchased from HyClone (GE Healthcare Life Sciences, Logan, UT, USA). The Annexin V-FITC apoptosis detection kit was purchased from Biomiga (Beijing, China). The cell proliferation detection kit was purchased from Promega Corp. (Madison, WI, USA). Acridine Orange/Ethidium Bromide (AO/EB), 4,6-Diamidino-2-phenylindole (DAPI), propidium iodide (PI) were purchased from Beijing Solarbio Science and Technology Co., Ltd., Beijing, China. Six-week-old BALB/c nude mice were purchased from Experimental Animal Center, Academy of Military Medical Sciences of PLA (Beijing, China) and were housed under standard pathogen-free conditions. The animal experimental protocols were approved by the Institutional Animal Care and Use Committee (IACUC) of the Chinese Academy of Military Medical Science (Changchun, China; 10ZDGG007). All surgeries were performed under sodium pentobarbital anesthesia, and all efforts were made to minimize suffering.

Plaque formation assay and cell proliferation assay. The A549 human lung adenocarcinoma cells were dispensed at $5 \times 10^{3}$ cells per well in a 96 -well plate at $37^{\circ} \mathrm{C}$ with $5 \% \mathrm{CO}_{2}$ for $24 \mathrm{~h}$. A549 cells were treated with $0.1,0.2,0.4$, and $0.8 \mu \mathrm{g}$ cisplatin, which determined the optimum does of cisplatin as $0.4 \mu \mathrm{g}$. The formation of virus plaques in A549 cells treated with ATV and cisplatin was measured via crystal violet staining. A549 cells were seeded in 12-well cell culture plates at $1 \times 10^{5}$ per well and cultured at $37^{\circ} \mathrm{C}$ with $5 \% \mathrm{CO}_{2}$ for $24 \mathrm{~h}$. The cells were then treated with ATV at 1 multiplicity of infection (MOI), $10 \mathrm{MOI}$, and $100 \mathrm{MOI}$ together with $0.4 \mu \mathrm{g}$ cisplatin. Along with Ad5-infected control wells, the infected cells were cultured at $37^{\circ} \mathrm{C}$ with $5 \% \mathrm{CO}_{2}$ for 24,48 , and $72 \mathrm{~h}$. The plaques that were stained by crystal violet were examined under an optical microscope. A549 cells were infected with either ATV $(1,10$, and $100 \mathrm{MOI})$ or a combination of ATV $(1,10$, and $100 \mathrm{MOI})$ and cisplatin $(0.4 \mu \mathrm{g})$. Cell viability was measured by the MTS cell proliferation kit at 24, 36, 48, 72, and $96 \mathrm{~h}$, following the manufacturer's instructions. Ad5-infected cells were used as negative controls.
Transwell migration and Matrigel invasion assay. Human A549 lung adenocarcinoma cells were dispensed at $5 \times 10^{4}$ cells per well in a 24 -well plate at $37^{\circ} \mathrm{C}$ with $5 \% \mathrm{CO}_{2}$ for $24 \mathrm{~h}$. The cells were infected with either ATV at an MOI of 10 and 100 or ATV at an MOI of 10 and 100 combined with $0.4 \mu \mathrm{g}$ cisplatin for 24 and $48 \mathrm{~h}$. The A549 cells were then seeded in the upper chamber of the cell culture inserts after trypsinization, and cultured for $24 \mathrm{~h}$. Cells that had migrated through the membrane were counted under a microscope after they were fixated by carbinol and stained with crystal violet. The experimental procedure of Matrigel invasion assay was the same as that for the Transwell migration assay except for incubation with Matrigel of the upper chamber for $1 \mathrm{~h}$ before seeding the cells. Ad5-infected cells were used as negative controls. And Transwell migration and Matrigel invasion assay was performed as described previously (24).

Xenograft and orthotopic tumor model BALB/c nude mice and their treatment strategy. The xenograft models were established via subcutaneous injection of A549 cells $\left(1 \times 10^{6} / 100 \mu \mathrm{l}\right)$ into the right legs of the mice. The orthotopic model was established via subcutaneous injection of A549 cells $\left(1 \times 10^{6} / 100 \mu \mathrm{l}\right)$ into the caudal vein. When the tumors had formed clearly (usually 4 days), the mice were divided randomly into five groups $(n=50)$. Group 1 was injected with $1 \times 10^{8}$ plaque forming units (PFU) of ATV in $100 \mu 1$ of phosphate-buffered saline (PBS). Group 2 was injected with $0.06 \mathrm{mg}$ of cisplatin in $100 \mu \mathrm{l}$ of PBS. Group 3 was injected with ATV $\left(1 \times 10^{8} \mathrm{PFU}\right)$ and cisplatin $(0.06 \mathrm{mg})$ in $100 \mu \mathrm{l}$ of PBS. Group 4 was injected with $1 \times 10^{8}$ PFU of Ad5 in $100 \mu 1$ of PBS. Group 5 was injected with $100 \mu$ of PBS. All groups were treated from the 0th day. The xenograft models were infected with ATV via intratumoral injection and treated with cisplatin via intraperitoneal injection, while the orthotopic models were infected with ATV via caudal vein injection and treated with cisplatin via intraperitoneal injection. Injections were given every four days for 16 days. The length and width of the xenograft tumors were measured every four days for 24 days using Vernier calipers. Survival of the xenograft and orthotopic mice models was observed every four days for 30 days. All the mice models were sacrificed at day 30 , and the xenografted tumors were removed and measured. Meanwhile, the lungs from the orthotopic models were removed and observed.

The analysis of A549 cells inhibition pathway by ATV. The key difference between apoptosis and cells death is the integrity of the cell membrane. Therefore, apoptosis and cells death could be obviously distinguished via DAPI, AO/EB and Annexin V staining. Human A549 lung adenocarcinoma cells were dispensed at $1 \times 10^{6}$ cells per well in a 6-well plate and incubated at $37^{\circ} \mathrm{C}$ with $5 \% \mathrm{CO}_{2}$ for $24 \mathrm{~h}$. The cells were then infected with 100 MOI ATV for $48 \mathrm{~h}$. The ATV-infected A549 cells were stained by $\mathrm{AO} / \mathrm{EB}$ and DAPI to confirm the inhibition pathway of A549 cells by ATV. The A549 cells (1x10 ${ }^{6}$ cells) were treated with $100 \mathrm{MOI}$ ATV for 24,48 , and $72 \mathrm{~h}$, and then the uninfected and ATV-infected A549 cells were harvested, washed with PBS three times, and incubated in the presence of $5 \mu \mathrm{l}$ Annexin V-FITC (early apoptotic marker appearing green) and $5 \mu \mathrm{l}$ of PI (late apoptotic marker appearing red) in $100 \mu \mathrm{l}$ of $1 \mathrm{X}$ binding buffer at room temperature in the dark 
A
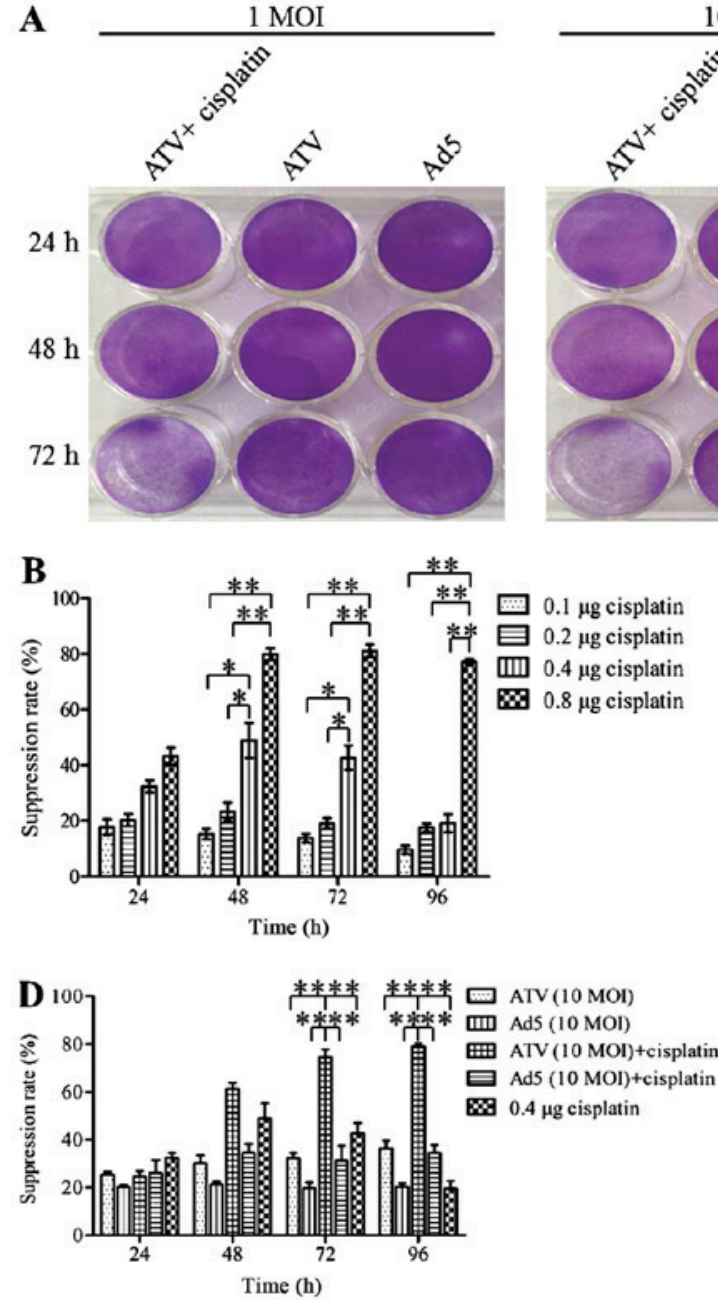

$10 \mathrm{MOI}$

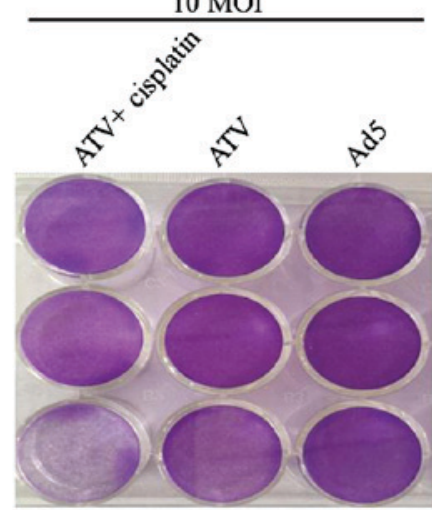

C
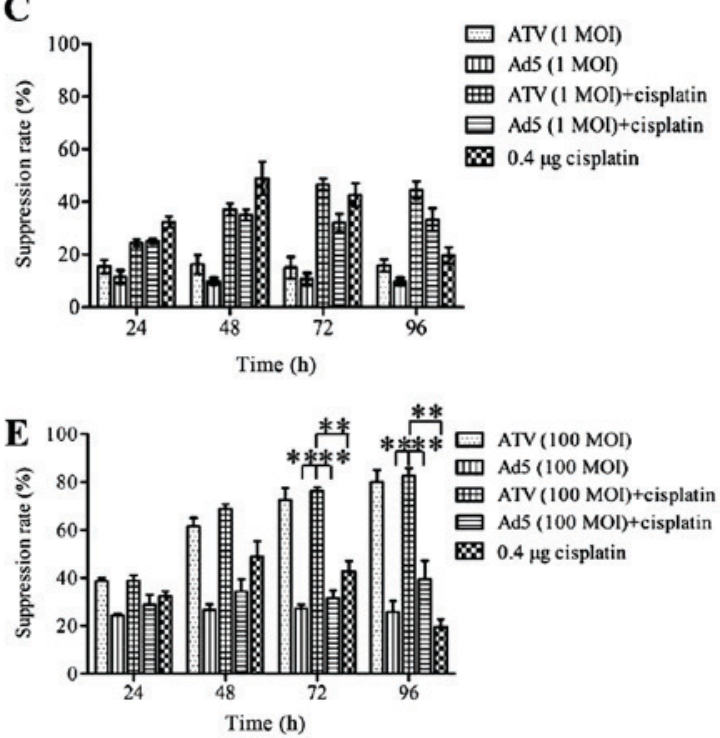

Figure 1. Plaque formation assay by crystal violet staining and cell proliferation assay by MTS. (A) A549 cells in 12 -well plates treated with 1 MOI, 10 MOI, and $100 \mathrm{MOI}$ of ATV and $0.4 \mu \mathrm{g}$ cisplatin for $24,48,72 \mathrm{~h}$ and then stained with $0.4 \%$ crystal violet after plaque phenotypes formed. (B) The suppression of proliferation of A549 cells treated by $0.1,0.2,0.4$, or $0.8 \mu \mathrm{g}$ of cisplatin. (C) The suppression of proliferation of A549 cells infected with 1 MOI ATV or a combination of the 1 MOI ATV and $0.4 \mu \mathrm{g}$ cisplatin. (D) The suppression of proliferation of A549 cells infected with $10 \mathrm{MOI}$ ATV or a combination of the $10 \mathrm{MOI}$ ATV and $0.4 \mu \mathrm{g}$ cisplatin. (E) The suppression of proliferation of A549 cells infected with $100 \mathrm{MOI}$ ATV or a combination of the $100 \mathrm{MOI}$ ATV and $0.4 \mu \mathrm{g}$ cisplatin. All values represent the mean $\pm \mathrm{SD}$. ${ }^{* *} \mathrm{P}<0.01$ and ${ }^{*} \mathrm{P}<0.05$. MOI, multiplicity of infection; $\mathrm{SD}$, standard deviation; Ad5, human adenovirus serotype 5.

for $15 \mathrm{~min}$, and then the apoptosis of A549 cells were analyzed by laser scanning confocal microscopy and flow cytometry. Ad5-infected cells were used as negative controls.

Statistical analysis. The statistical analysis was performed using data from at least three independent experiments using the Statistical Package for the Social Sciences (SPSS) statistical software package (version 15.0; SPSS, Inc., Chicago, IL, USA), and the results were obtained using GraphPad Prism version 7.0 (GraphPad Software, Inc., La Jolla, CA, USA). Student's t-test or one-way analysis of variance followed by Tukey's post hoc test were used. Differences with a $\mathrm{P}<0.05$ or $\mathrm{P}<0.01$ were considered to indicate a statistically significant difference. Data are presented as the mean \pm standard deviation (SD) values.

\section{Results}

Proliferation inhibition effect of A549 cells. The results for the inhibition of A549 cell proliferation are shown in Fig. 1A-E. The plaque formation assay is shown in Fig. 1A. The MTS assays showed that the proliferation of A549 cells was suppressed significantly at 24,48 and $72 \mathrm{~h}$ by $0.8 \mu \mathrm{g}$ cisplatin $(\mathrm{P}<0.01)$. However, this inhibitory effect may be caused by itself toxic. The proliferation of A549 cells was suppressed significantly at 24 and $48 \mathrm{~h}$ by $0.4 \mu \mathrm{g}$ cisplatin $(\mathrm{P}<0.05)$ and the inhibitory effect of A549 cells in $0.4,0.1$ and $0.2 \mu \mathrm{g}$ cisplatin had no significant difference $(\mathrm{P}>0.05)$. This result indicated that the inhibitory effect of $0.4 \mu \mathrm{g}$ was not induced by itself toxic. Therefore the optimum dose of cisplatin was $0.4 \mu \mathrm{g}$. The growth of A549 cells could be suppressed by ATV or ATV combined with cisplatin in a dose- and time-dependent manner. The inhibition rate of A549 cells was increased significantly by $0.4 \mu \mathrm{g}(\mathrm{P}<0.05)$ and $0.8 \mu \mathrm{g}(\mathrm{P}<0.01)$ cisplatin compared to 0.1 and $0.2 \mu \mathrm{g}$ cisplatin, while the higher toxicity of $0.8 \mu \mathrm{g}$ cisplatin made it unfit for subsequent experiments (Fig. 1B). The growth suppression of A549 cells was not increased by 1 MOI ATV. However, increased growth suppression of A549 cells was observed by $1 \mathrm{MOI}$ ATV and $0.4 \mu \mathrm{g}$ cisplatin, which reached a peak of 3.11-fold greater than that of the $1 \mathrm{MOI}$ ATV-infected cells at $72 \mathrm{~h}$ (Fig. 1C). The growth suppression induced by 10 MOI ATV and $0.4 \mu \mathrm{g}$ cisplatin was significantly 
A

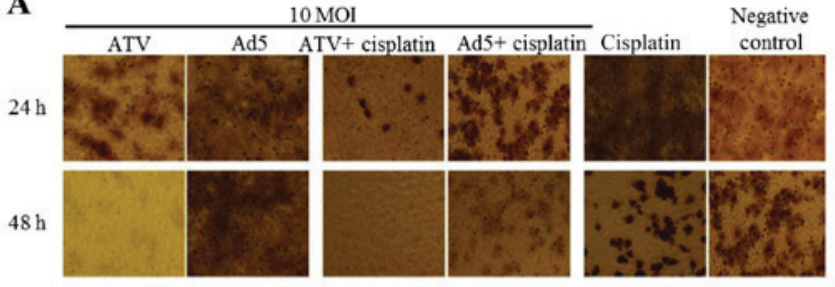

B

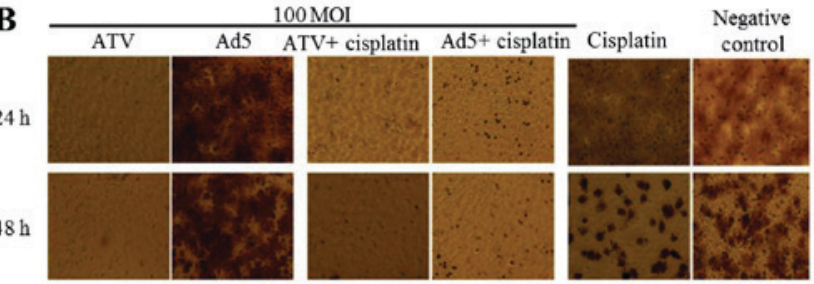

C

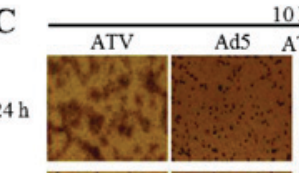

$48 \mathrm{~h}$
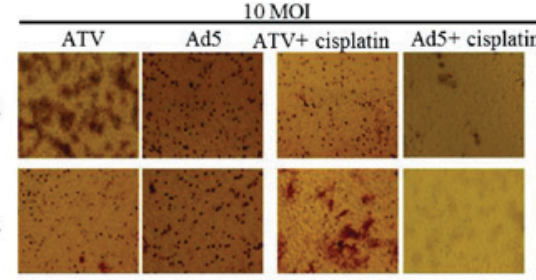

D

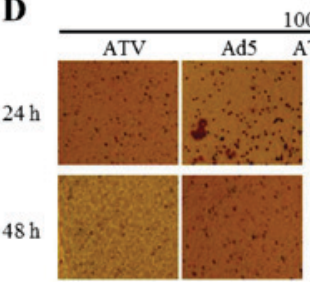

$100 \mathrm{MOI}$

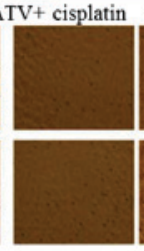

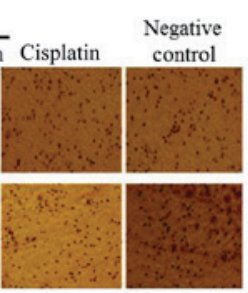
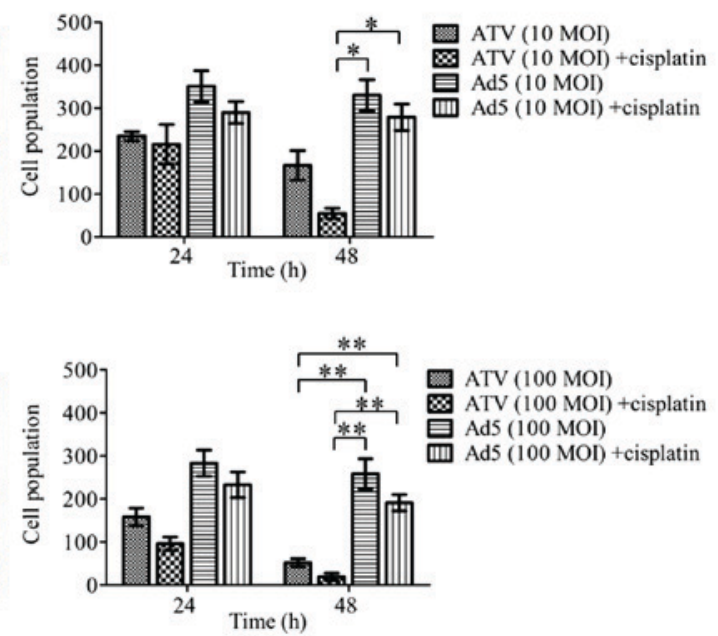

Figure 2. Migration and invasion suppression effects on A549 cells by the Transwell assay. (A) The migration suppression of 10 MOI ATV-infected A549 cells and A549 cells treated with 10 MOI ATV-and $0.4 \mu \mathrm{g}$ cisplatin. (B) The migration suppression of A549 cells infected with 100 MOI ATV-infected in cells treated with the combination of 100 ATV- and $0.4 \mu \mathrm{g}$ cisplatin. (C) The invasion suppression of A549 cells infected with 10 MOI ATV and in those treated with the combination of 10 MOI ATV- and $0.4 \mu \mathrm{g}$ cisplatin. (D) The invasion suppression of A549 cells infected with 10 MOI ATV and in those treated with the combination of 100 MOI ATV-and $0.4 \mu \mathrm{g}$ cisplatin. All values represent the mean $\pm \mathrm{SD}$. ${ }^{* *} \mathrm{P}<0.01$ and ${ }^{*} \mathrm{P}<0.05$ (magnification, $\mathrm{x} 100$ ). MOI, multiplicity of infection; SD, standard deviation; Ad5, human adenovirus serotype 5.

higher than that of the control group $(\mathrm{P}<0.01)$. Meanwhile, the combination of $10 \mathrm{MOI}$ and $0.4 \mu \mathrm{g}$ cisplatin caused increasing growth suppression from 24 to $72 \mathrm{~h}$, which reached a peak of 2.5-fold greater than that of the 10 MOI ATV-infected cells at $72 \mathrm{~h}$ (Fig. 1D). The growth suppression rate of $100 \mathrm{MOI}$ ATV infected-cells was also significantly higher than that of the control group $(\mathrm{P}<0.01)$. However, the growth suppression was not significantly different between the combination of 100 MOI ATV 100 with $0.4 \mu \mathrm{g}$ and 100 MOI ATV $(\mathrm{P}>0.05)$ (Fig. 1E). Therefore, the combination of ATV at an MOI of 10 or 100 with $0.4 \mu \mathrm{g}$ cisplatin showed a synergistic effect on the inhibition of the growth of A549 cells.

Migration and invasion suppression effect of A549 cells. The results of A549 cell migration and Matrigel invasion are shown in Fig. 2A-B. The migration inhibition effect of $10 \mathrm{MOI}$ ATV and $0.4 \mu \mathrm{g}$ cisplatin-treatment A549 cells for $48 \mathrm{~h}$ was significantly higher than those of the other groups at $48 \mathrm{~h}(\mathrm{P}<0.05)$ (Fig. 2A). The migration inhibition effects of 100 MOI ATV-infected A549 cells and the combination of $100 \mathrm{MOI}$ ATV- with $0.4 \mu \mathrm{g}$ cisplatin-treatment A549 cells showed no significant differences, but were significantly higher than those of the control groups at $48 \mathrm{~h}(\mathrm{P}<0.01)$ (Fig. $2 \mathrm{~B})$. The Matrigel invasion assay showed similar results to the migration assay (Fig. 2C-D).

Tumor growth inhibition and survival rate of $B A L B / c$ nude mice model. The results of the tumor growth inhibition experiment are shown in Fig. 3A and the tumor growth inhibition of ATV and cisplatin group was significantly lower than that of the PBS group $(\mathrm{P}<0.01)$. The results of for the survival rate over 30 days are shown in Fig. 3B-C. The photographs of the subcutaneous tumors and lungs from the orthotopic models excised from the nude mice are shown in Fig. 3D. The inhibition of tumor growth in the treatment group was higher than those of the other groups. Although the comparison between treatment groups showed no significant differences in terms of tumor growth inhibition, the survival rate of the combination group was significantly higher than that of the cisplatin group and indicated that the toxicity of cisplatin could be reduced by combination of ATV and cisplatin. 
A

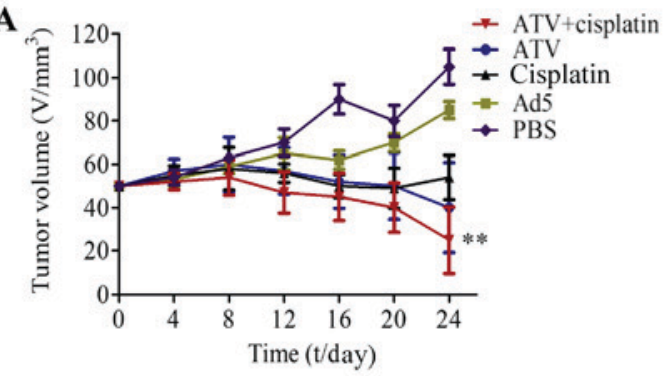

B

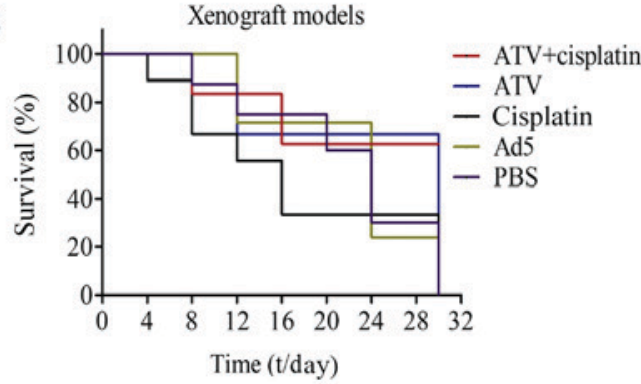

C

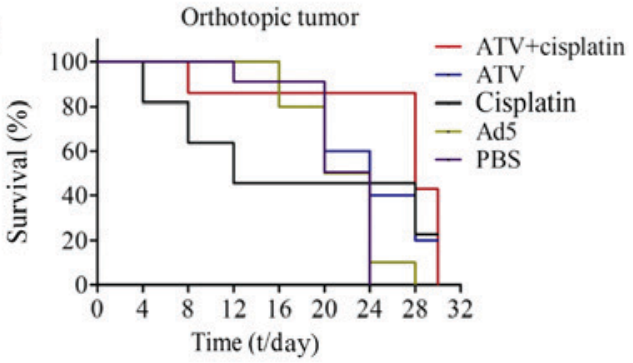

D

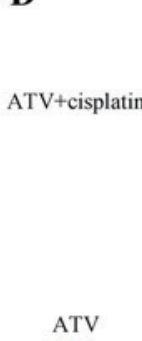

Ad5

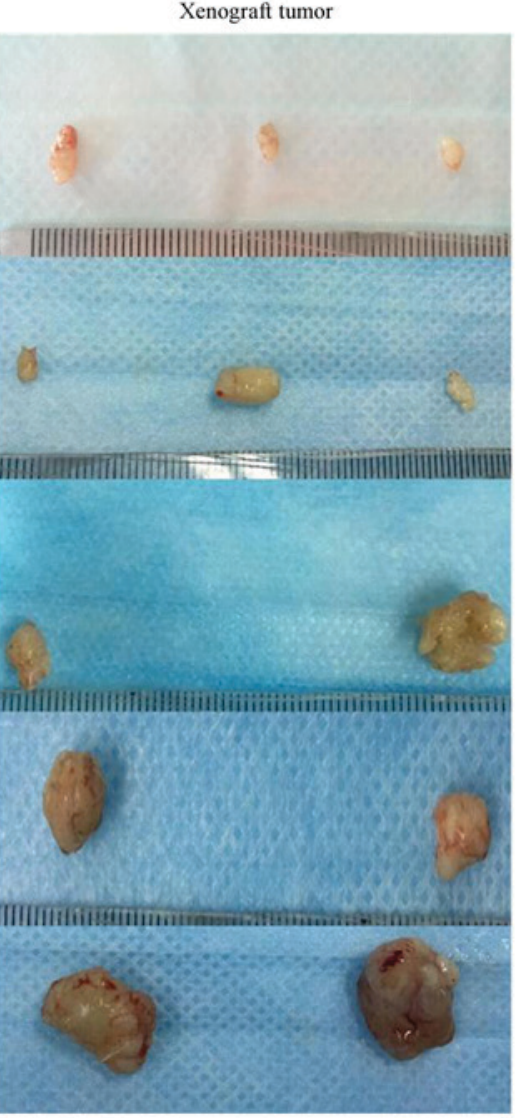

Orthotopic tumor

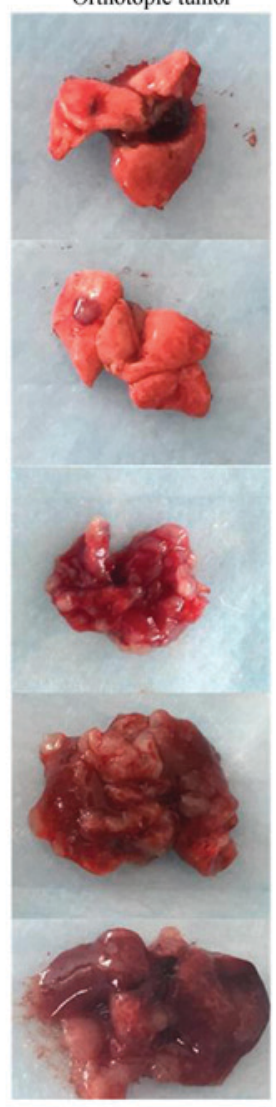

Figure 3. The tumor growth inhibition and survival rate of BALB/c nude mice transplanted with A549 cells. (A) The tumor growth inhibition effect in transplanted nude mice. The tumor volume of the ATV and cisplatin group was significantly lower than that of the PBS group (P<0.01). (B) The survival rate of xenograft tumor nude mice. (C) The survival rate of orthotopic tumor nude mice models. (D) The xenografted tumors were removed and measured at the 30 th day and the lungs from orthotopic tumor nude mice models were removed on the 28 th day. All values represent the mean \pm SD. ${ }^{* *} \mathrm{P}<0.01$ vs. PBS group. PBS, phosphate-buffered saline; MOI, multiplicity of infection; SD, standard deviation; Ad5, human adenovirus serotype 5.

Mechanism of action of ATV on A549 cells. The results of the apoptosis detection assays of A549 cells are shown in Fig. 4A-F. The results of AO/EB (Fig. 4A) and DAPI (Fig. 4B) staining proved that ATV induced apoptosis in the A549 cells. The Annexin V-FITC and PI double-labeled positive cells could be observed among the ATV-infected A549 cells under the laser scanning confocal microscope (Fig. 4C), which indicated that apoptotic morphological characteristics of the A549 cells was caused by apoptin expressed from ATV. The degree of apoptosis in the ATV-infected A549 cells at various time-points was evaluated under flow cytometry (Fig. 4D-F), which showed that the apoptosis rate of ATV-infected A549 cells at $48 \mathrm{~h}$ was 3.8-fold greater than that of ATV-infected A549 cells at $24 \mathrm{~h}(\mathrm{P}<0.05)$; however, there was no further significant increase from 48 to $72 \mathrm{~h}$. The trend in the variation of the death rate of ATV-infected A549 cells was the same as the apoptosis rate. Thus, in A549 cells, ATV induced mainly apoptosis.

\section{Discussion}

Cancer cells can be recognized and killed specifically by oncolytic viruses. Many clinical studies have demonstrated the good antitumor activity of oncolytic viruses (25-28). Oncolytic viruses have been used as delivery vectors that carry specific therapeutic proteins to destroy malignant cells during replication. Thus, oncolytic viruses have emerged as important methods in cancer treatment. Adenovirus, herpes virus, and orthopoxviruse, as oncolytic virus vehicles, have been applied successfully to antitumor therapy. Apoptin is considered as one of the most promising antitumor proteins because it induces specific apoptosis of cancer cells without effecting normal cells. Oncolytic viruses expressing apoptin proteins have been constructed and tested, including a dual cancer-specific oncolytic adenovirus containing apoptin, poly A, hTERTp, and E1a. Experiments have proved that tumor cell growth could be suppressed significantly by a dual cancer-specific oncolytic adenovirus both in vitro and in vivo (19-22).

Currently, chemotherapy, radiotherapy, and biotherapy are the main strategies for cancer treatment. However, because of the specificity of tumors and the side effects of these methods, treatment is far from satisfactory. With the emergence of, for example, drug resistance (3) and DNA damage repair $(29,30)$ during the treatment, the therapeutic effect is compromised in cancers when using monotherapy. Therefore, it is necessary to use combinations of two or more therapeutic methods that act via different mechanisms to produce synergistic effects to treat cancers. The ideal combination treatment should reduce toxicity and increase the therapeutic effect. Some clinical 
A
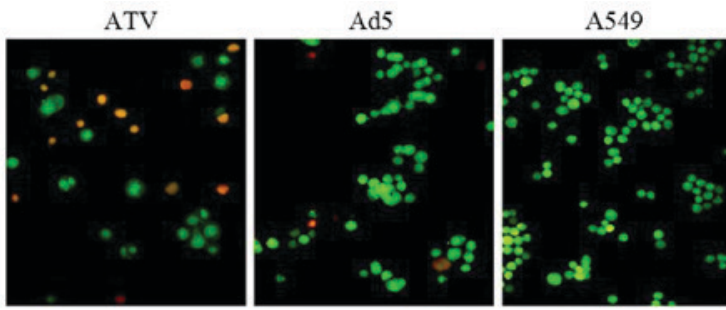

$\mathbf{C}$
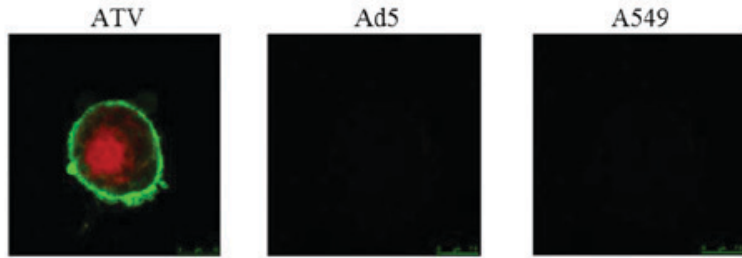

D

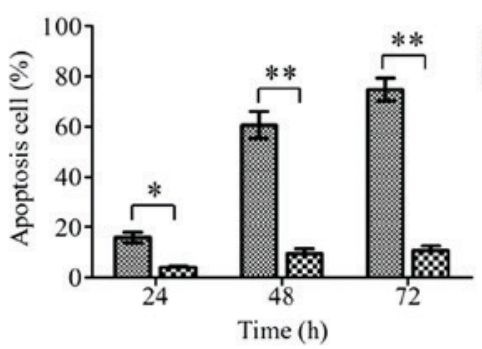

ATV

Ad5

E

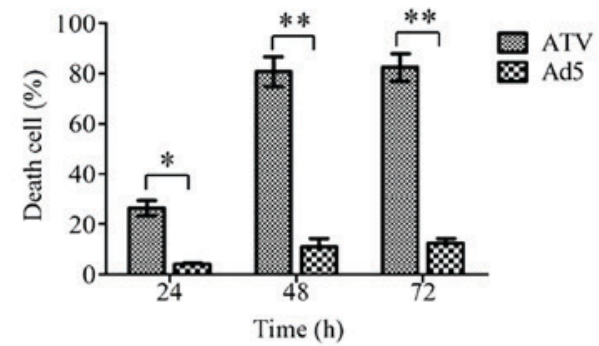

B
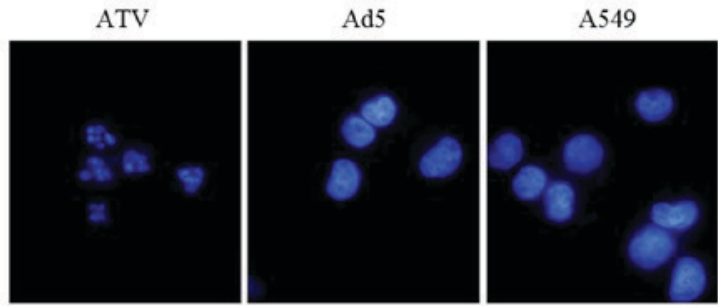

F
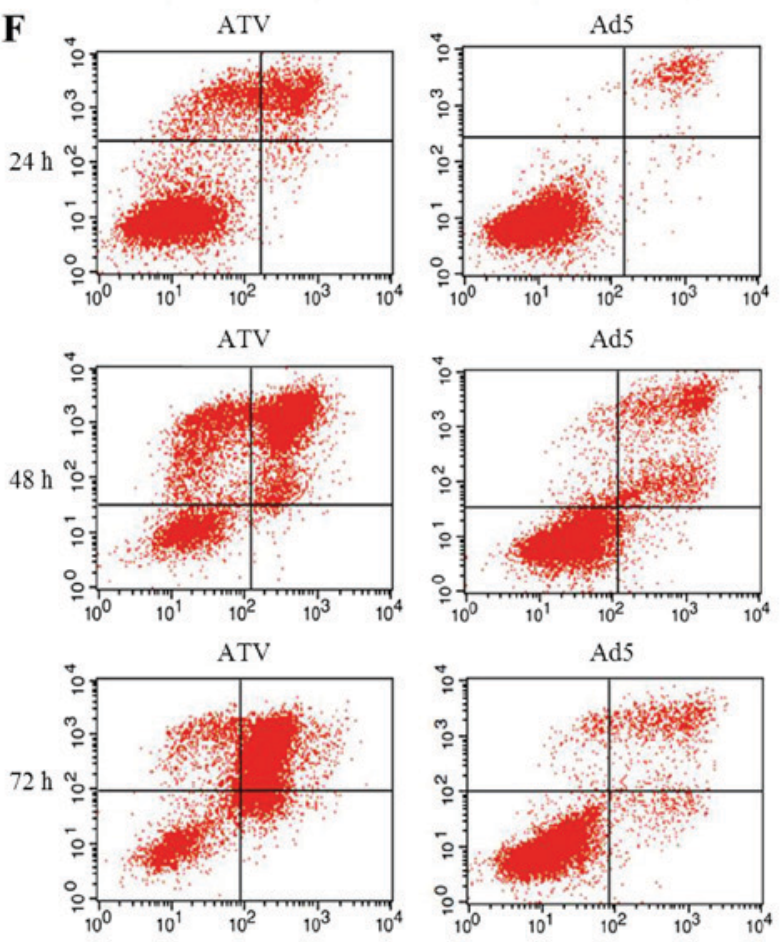

Figure 4. The mode of action of ATV on A549 cells. (A) AO/EB staining of ATV-infected A549 cells (magnification, x100). (B) DAPI staining of ATV-infected A549 cells (magnification, x200). (C) Apoptosis assay of ATV-infected A549 cells by laser scanning confocal microscope (magnification, x400). (D) The apoptosis rate of ATV-infected A549 cells at 24, 48 and 72 h. (E) The death rate of ATV-infected A549 cells at 24, 48 , and 72 h. (F) Apoptosis assay of ATV-infected A549 cells by flow cytometry. All values represent the mean \pm SD. ${ }^{* *} \mathrm{P}<0.01$ and ${ }^{*} \mathrm{P}<0.05$. AO/EB, Acridine Orange/Ethidium Bromide; DAPI, 4,6-Diamidino-2-phenylindole; SD, standard deviation; Ad5, human adenovirus serotype 5.

studies have shown that a good antitumor ability, reduced toxicity, and increased treatment effect could be exerted by combining oncolytic adenovirus with a chemotherapy drug such as cisplatin $(17,18)$.

In this study, we developed a synergistic antitumor strategy, which combined a dual cancer-specific oncolytic adenovirus (ATV) with cisplatin. We confirmed the enhanced antitumor and reduced toxicity of ATV with cisplatin in A549 cells in vitro and in vivo. In our in vitro experiments, the MTS assays were used to detect the suppression of the ATV, Ad5 and cisplatin in A549 cells. We found that $0.8 \mu \mathrm{g}$ cisplatin significantly inhibited A549 cells proliferation at 48,72 and $96 \mathrm{~h}$, while $0.4 \mu \mathrm{g}$ cisplatin significantly inhibited A549 cells proliferation at 48 and $72 \mathrm{~h}$, however, considering the relationship between toxicity and efficacy of cisplatin, we finally determined the dosage of cisplatin was $0.4 \mu \mathrm{g}$. The ATV and cisplatin combination group have no significant difference with ATV alone group, but significantly higher than the other experimental groups. In addition, 10 MOI ATV and cisplatin combination group can significantly inhibit the proliferation of A549 cells, the inhibitory effect was significantly higher than the other experimental groups. These results show that combined application of ATV and cisplatin can effectively improve the suppression effect of the tumor.

In our in vivo tumor model experiments in nude mice, compared with the PBS control group and the Ad5 control group use cisplatin alone can significantly inhibit the growth of the tumor and prolong the survival time of nude mice, but due to its toxicity, it can lead to the death of individual nude mice in the early stages of treatment, and the result also appeared in the Ad5 and cisplatin combination group. In ATV and cisplatin combination group, the combined application of ATV and cisplatin can obviously inhibit the growth of tumor, and prolong the survival time of nude mice, while avoiding the death of individual nude mice due to toxicity of cisplatin in the early stages of treatment. This result suggests that cisplatin combined with Ad5 can not reduce the toxicity of cisplatin, but combination of cisplatin and the dual cancer-specific oncolytic adenovirus (ATV) can effectively reduce the toxicity of cisplatin, improve the survival rate of nude mice in the early stages of treatment. In the metastatic tumor model, 
through comparing the size of the isolated tumor volume, it is obvious that although the tumor volume of ATV and cisplatin combination group has no significant difference with the tumor volume of ATV alone group, but it is much smaller than that of the other experimental groups. In the in-situ tumor model, uncancerous lungs were isolated from nude mice in the ATV and cisplatin combination groups, and there was a slight canceration of the lungs in ATV alone group. In the remaining groups, the lungs of nude mice had become cancerous, the lung tissue was swollen and congested and lost the original form, suggesting that the combined application of ATV and cisplatin was better than that of ATV or cisplatin alone. In the experiments in mechanism of action of ATV on A549 cells, the characteristic of apoptosis of A549 cells is that integrity of the cell membrane does not damage and the cell nuclear membrane and nucleolus fragmentations has been appeared. Cell nucleus state could be obviously observed by DAPI staining. After AO/EB staining, bright green fluorescence in early apoptotic cells, orange fluorescence in late apoptotic cells and red fluorescence in death cells could be observed under a fluorescence microscope. The cytomembrane of apoptotic cells could be dyed green and the nuclear of apoptotic cells could be dyed red after Annexin V staining. The apoptosis rate and death rate of A549 cells were almost the same at 24, 48 and $72 \mathrm{~h}$. The present study showed that the death of A549 cells was mainly induced by apoptosis. These results showed that ATV inhibits the proliferation of A549 cells by inducing apoptosis. Experimental results show that combined application of ATV and cisplatin can effectively reduce the toxicity of cisplatin and can improve the therapeutic effect of the tumor. The results demonstrated a promising therapeutic potential for this combined antitumor drug formulation and provided a foundation for future preclinical studies of antitumor treatment.

\section{Acknowledgements}

Not applicable.

\section{Funding}

The present study was supported by the National Key Research and Development Program of China (grant no. 2016YFC1200900), the Major Technological Program of Changchun City (grant no. 16ss11), the National Science and Technology Major Project (Major New Drugs Innovation and Development) (grant no. 2018ZX09301053-004-001) and the Shandong Provincial Key Research and Development Program (grant no. 2016CYJS01A03).

\section{Availability of data and materials}

All datasets generated or analyzed during this current study are included in this published article.

\section{Authors' contributions}

$\mathrm{XL}$ and NJ conceived and designed the experiments. JJ, YZ, FS, ZC, SC, YL, WL, XY, ML and CC performed the experiments. YC, SL, JZ and GY analyzed the data. YL, NJ and
YZ wrote the paper. All authors read and approved the final manuscript.

\section{Ethics approval and consent to participate}

The animal experimental protocols were approved by the IACUC of the Chinese Academy of Military Medical Science (10ZDGG007). All surgeries were performed under sodium pentobarbital anesthesia, and all efforts were made to minimize suffering.

\section{Patient consent for publication}

Not applicable.

\section{Competing interests}

The authors declare that they have no competing interests.

\section{References}

1. Chen W, Zheng R, Zhang S, Zeng H, Zou X and He J: Report of Cancer Incidence and Mortality in China, 2013. China Cancer 25: 1-8 2017 (In Chinese).

2. Min YC: The development in the study of platinum-based antineoplastic drugs. Chin J Mod Drug Appl 9: 282, 2015.

3. Brabec V and Kasparkova J: Molecular aspects of resistance to antitumor platinum drugs. Drug Resist Updat 5: 147-161, 2002.

4. Arany I and Safirstein RL: Cisplatin nephrotoxicity. Semin Nephrol 23: 460-464, 2003.

5. Ali BH and Al Moundhri MS: Agents ameliorating or augmenting the nephrotoxicity of cisplatin and other platinum compounds: A review of some recent research. Food Chem Toxicol 44: 1173-1183, 2006.

6. Christova TY, Gorneva GA, Taxirov SI, Duridanova DB and Setchenska MS: Effect of cisplatin and cobalt chloride on antioxidant enzymes in the livers of Lewis lung carcinoma-bearing mice: Protective role of heme oxygenase. Toxicol Lett 138: 235-242, 2003.

7. Spracklen TF, Vorster AA, Ramma L, Dalvie S and Ramesar RS: Promoter region variation in NFE2L2 influences susceptibility to ototoxicity in patients exposed to high cumulative doses of cisplatin. Pharmacogenomics J 17: 515-520, 2017.

8. Maddika S, Mendoza FJ, Hauff K, Zamzow CR, Paranjothy T and Los M: Cancer-selective therapy of the future: Apoptin and its mechanism of action. Cancer Biol Ther 5: 10-19, 2006.

9. Leliveld SR, Dame RT, Mommaas MA, Koerten HK, Wyman C, Danen-van Oorschot AA, Rohn JL, Noteborn MH and Abrahams JP: Apoptin protein multimers form distinct higher-order nucleoprotein complexes with DNA. Nucleic Acids Res 31: 4805-4813, 2003

10. Zhong X, Zhao H, Liang S, Zhou D, Zhang W and Yuan L: Gene delivery of apoptin-derived peptide using an adeno-associated virus vector inhibits glioma and prolongs animal survival. Biochem Biophys Res Commun 482: 506-513, 2017.

11. Zhang J, Hou L, Wu X, Zhao D, Wang Z, Hu H, Fu Y and He J: Inhibitory effect of genetically engineered mesenchymal stem cells with Apoptin on hepatoma cells in vitro and in vivo. Mol Cell Biochem 416: 193-203, 2016.

12. Gupta SK, Tiwari AK, Gandham RK and Sahoo AP: Combined administration of the apoptin gene and poly (I:C) induces potent anti-tumor immune response and inhibits growth of mouse mammary tumors. Int Immunopharmacol 35: 163-173, 2016.

13. Vragniau C, Hubner JM, Beidler P, Gil S, Saydaminova K, Lu ZZ, Yumul R, Wang H, Richter M, Sova P, et al: Studies on the interaction of tumor-derived HD5 alpha defensins with adenoviruses and implications for oncolytic adenovirus therapy. J Virol 91: pii: e02030-16, 2017.

14. Mato-Berciano A, Raimondi G, Maliandi MV, Alemany R, Montoliu L and Fillat C: A NOTCH-sensitive uPAR-regulated oncolytic adenovirus effectively suppresses pancreatic tumor growth and triggers synergistic anticancer effects with gemcitabine and nab-paclitaxel. Oncotarget 8: 22700-22715, 2017. 
15. Li X, Wang P, Li H, Du X, Liu M, Huang Q, Wang Y and Wang S: The efficacy of oncolytic adenovirus is mediated by T-cell responses against virus and tumor in syrian hamster model. Clin Cancer Res 23: 239-249, 2017.

16. Kim SY, Kang D, Choi HJ, Joo Y, Kim JH and Song JJ: Prime-boost immunization by both DNA vaccine and oncolytic adenovirus expressing GM-CSF and shRNA of TGF- $\beta 2$ induces anti-tumor immune activation. Oncotarget 8: 15858-15877, 2017.

17. Wang S, Shu J, Chen L, Chen X, Zhao J, Li S, Mou X and Tong X: Synergistic suppression effect on tumor growth of ovarian cancer by combining cisplatin with a manganese superoxide dismutase-armed oncolytic adenovirus. Onco Targets Ther 9: 6381-6388, 2016.

18. Ma B, Wang Y, Zhou X, Huang P, Zhang R, Liu T, Cui C, Liu X and Wang Y: Synergistic suppression effect on tumor growth of hepatocellular carcinoma by combining oncolytic adenovirus carrying XAF1 with cisplatin. J Cancer Res Clin Oncol 141: 419-429, 2015

19. Qi Y, Guo H, Hu N, He D, Zhang S, Chu Y, Huang Y, Li X, Sun L and Jin N: Preclinical pharmacology and toxicology study of Ad-hTERT-Ela-Apoptin, a novel dual cancer-specific oncolytic adenovirus. Toxicol Appl Pharmacol 280: 362-369, 2014.

20. Zhang M, Wang J, Li C, Hu N, Wang K, Ji H, He D, Quan C, Li X, Jin N and Li Y: Potent growth-inhibitory effect of a dual cancer-specific oncolytic adenovirus expressing apoptin on prostate carcinoma. Int J Oncol 42: 1052-1060, 2013.

21. Liu L, Wu W, Zhu G, Liu L, Guan G, Li X, Jin N and Chi B: Therapeutic efficacy of an hTERT promoter-driven oncolytic adenovirus that expresses apoptin in gastric carcinoma. Int J Mol Med 30: 747-754, 2012.

22. Li X, Liu Y, Wen Z, Li C, Lu H, Tian M, Jin K, Sun L, Gao P, Yang E, et al: Potent anti-tumor effects of a dual specific oncolytic adenovirus expressing apoptin in vitro and in vivo. Mol Cancer 9: 10, 2010.
23. Yin XZ, Chen S, Li WJ, Zhu YL, Li YQ, Cui CX, Li M, Cui YL, Zhao J, Li SZ et al: Inhibitory effect of apopotin-loaded oncolytic adenovirus ATV on human cervical carcinoma HeLa cells. Chin J Cancer Biother 24: 1356-1361, 2017 (In Chinese).

24. Wang X, Xu L, Wu Q, Liu M, Tang F, Cai Y, Fan W, Huang H and $\mathrm{Gu} \mathrm{X}$ : Inhibition of LDHA deliver potential anticancer performance in renal cell carcinoma. Urol Int 99: 237-244, 2017.

25. Robinson S and Galanis E: Potential and clinical translation of oncolytic measles viruses. Expert Opin Biol Ther 17: 353-363, 2017.

26. Ungerechts G, Bossow S, Leuchs B, Holm PS, Rommelaere J, Coffey M, Coffin R, Bell J and Nettelbeck DM: Moving oncolytic viruses into the clinic: Clinical-grade production, purification, and characterization of diverse oncolytic viruses. Mol Ther Methods Clin Dev 3: 16018, 2016.

27. Patil S, Rao RS and Majumdar B: Clinical trials with oncolytic viruses: Current and future prospects. J Contemp Dent Pract 16: i-ii, 2015.

28. Burke J, Nieva J, Borad MJ and Breitbach CJ: Oncolytic viruses: Perspectives on clinical development. Curr Opin Virol 13: 55-60, 2015.

29. Štefančíková L, Lacombe S, Salado D, Porcel E, Pagáčová E, Tillement O, Lux F, Depeš D, Kozubek S and Falk M: Effect of gadolinium-based nanoparticles on nuclear DNA damage and repair in glioblastoma tumor cells. J Nanobiotechnology 14: 63, 2016.

30. Sengupta S, Mantha AK, Song H, Roychoudhury S, Nath S, Ray S and Bhakat KK: Elevated level of acetylation of APE1 in tumor cells modulates DNA damage repair. Oncotarget 7: 75197-75209, 2016.

C) (ㅇ) This work is licensed under a Creative Commons cc) ${ }_{\text {EY NO ND }}$ Attribution-NonCommercial-NoDerivatives 4.0 International (CC BY-NC-ND 4.0) License. 